\section{Die Rhinitis-Asthma-Connection}

\author{
Sowohl allergische als auch nicht-allergische Rhinitis sind mit einer \\ erhöhten Asthmaprävalenz assoziiert. Ein dänisches Forscherteam \\ ging jetzt den Pathomechanismen nach, die dieser Beobachtung \\ zugrunde liegen.
}

$\mathrm{n}$ eine Analyse der Asthmaprävalenz bei allergischer und nicht-allergischer Rhinitis wurden 290 siebenjährige Kinder der dänischen Geburtskohortenstudie COPSAC (Copenhagen Prospective Study on Asthma in Childhood) einbezogen, 38 hatten eine allergische Rhinitis, 67 eine nicht-allergische Rhinitis und 185 dienten als Kontrolle.

Kinder mit allergischer Rhinitis zeigten im Vergleich zu den asymptomatischen Kontrollen erhöhte Prävalenzen für Asthma (21 vs. 5\%, p =0,002), Nahrungsmittelsensibilisierungen (47 vs. $13 \%, \mathrm{p}<0,001$ ) und Ekzeme (66 vs. $43 \%, \mathrm{p}=0,01)$, erhöhtes Gesamt-IgE (155 vs. $41 \mathrm{kU} / \mathrm{l}, \mathrm{p}<0,001)$, erhöhte Blut-Eosinophilenzahl $\left(0,46 \times 10^{9} / 1\right.$ vs. $\left.0,30 \times 10^{9} / \mathrm{l}, \mathrm{p}=0,01\right)$, Stickstoffmonoxid in der Ausatemluft (FeNO; 15,9 vs. $6,6 \mathrm{ppb}, \mathrm{p}<0,001)$ und bronchiale $\mathrm{Hy}-$ perreagibilität ( 23 vs. $9 \%, p=0,008$ ). Auch war die allergische Rhinitis mit einer erhöhten Filaggrin-Null-Mutations- rate assoziiert (Odds Ratio 3,3, 95\%-Konfidenzintervall 1,3 bis 8,3 ). Bei den Kindern mit nicht-allergischer Rhinitis fand sich ebenfalls eine erhöhte Asthmaprävalenz ( $20 \%$ vs. $5 \%$, p = 0,001), aber kein Zusammenhang mit Filaggrin-Null-Mutationen, Ekzemen, totalem IgE, FeNO oder bronchialer Hyperreagibilität.

Fazit: Bei Kindern sind allergische und nicht-allergische Rhinitis mit einer erhöhten Asthmaprävalenz assoziiert. Assoziationen mit allergischen oder entzündlichen Begleitparametern finden sich aber nur bei den Kindern mit allergischer Rhinitis.

$b k$

Chawes BLK et al. Children with allergic and nonallergic rhinitis have a similar risk of asthma. J Allergy Clin Immunol 2010; 126: $567-73$

\title{
Milbenasthma: Add-on-SCIT spart Glukokortikoide
}

\section{Vorrangiges Ziel der Asthmatherapie ist die Asthmakontrolle. Um die dazu nötige Dosis inhalativer Glukokortikoide gerade für Kinder mög- lichst gering zu halten, werden steroidsparende Add-on-Therapien wie eine spezifische Immuntherapie interessant.}

$\mathrm{n}$ einer neuen kontrollierten Multizenterstudie wurde die Wirksamkeit einer subkutanen spezifischen Immuntherapie (SCIT) als Add-on-Therapie bei Kindern mit einem hochdosierten hypoallergenen Extrakt aus Dermatophagoides pteronyssinus überprüft. Im Rahmen dieser aus ethischen Gründen offen durchgeführten Studie erhielten 65 Kinder und Jugendliche mit milbenassoziiertem, leichtem bis moderatem Asthma der GINA-Schweregrade II und III nach stabiler Einstellung auf inhalatives Fluticasonpropionat (FP) randomisiert entweder subkutan ein Milbenallergoid plus FP oder FP alleine. Wäh-

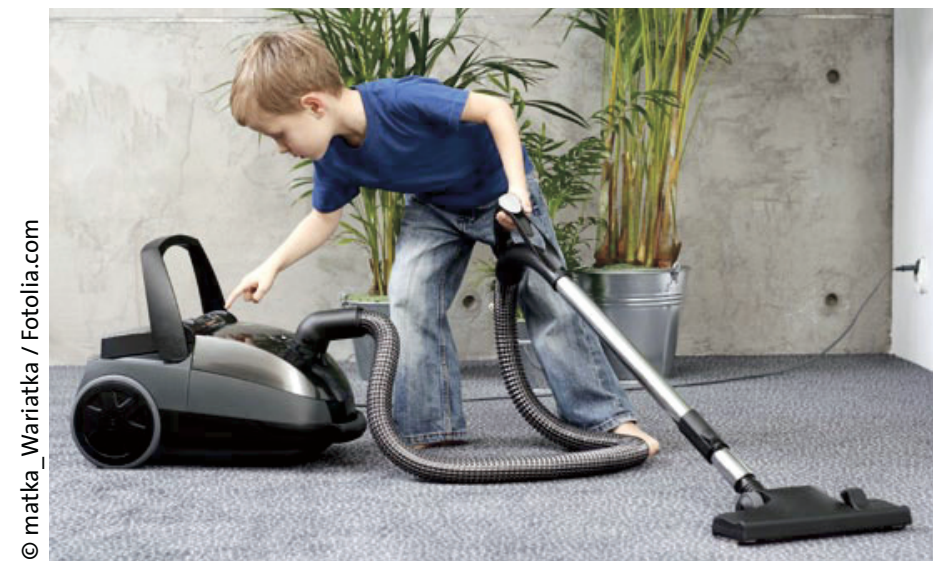

Die Kontrolle von persistierendem Asthma als Reaktion auf Hausstaubmilben soll für Kinder keine unnötige Zusatzbelastung sein unterstützen kann eine Addon-SCIT. rend zwei fünfmonatigen Winterperioden wurde die Glukokortikoidtherapie in prädefinierten Reduktionsschritten auf die niedrigste zur Asthmakontrolle notwendige Dosis adjustiert.

Dabei konnten bei den Kindern unter FP plus subkutan injiziertem Milbenallergoid mehr Dosisreduktionsschritte vorgenommen werden als bei Kindern unter alleiniger Pharmakotherapie. Die durchschnittliche Tagesdosis in der SCIT-FP-Gruppe reduzierte sich von $330,3 \mu \mathrm{g}$ auf $151,5 \mu \mathrm{g}$ nach zwei Behandlungsjahren, in der Kontrollgruppe von 290,6 $\mu \mathrm{g}$ auf 206,3 $\mu \mathrm{g}$. Verglichen mit der Kontrollgruppe verbesserte sich auch die morgendliche Atemstromstärke $(p=0,0315)$, die Spiegel von spezifischen IgG1 und IgG4 stiegen signifikant stärker an $(\mathrm{p}=0,0001$ und $\mathrm{p}<0,0001)$.

Fazit: Eine subkutane MilbenallergoidTherapie, gegeben über zwei Jahre, hat bei Kindern und Jugendlichen mit milbenassoziiertem Asthma einen deutlichen Glukokortikoid-sparenden Effekt.

Zielen $\mathbf{S}$ et al. Steroid-sparing effects with allergen-specific immunotherapy in children with asthma: a randomized controlled trial. J Allergy Clin Immunol 2010; 126: 942-9 